\title{
MacCallum's Triangle - Is It Rheumatic? Is It Traumatic? Or Is It Both?
}

\section{S. Siew}

Division of Human Pathology, College of Osteopathic Medicine, Michigan State University, East Lansing, MI, USA

Rheumatic carditis is a pancarditis, however, the parietal endocardium is affected to the least obvious degree. In 1924, MacCallum [1] described, in acute rheumatic fever, involvement of the endocardium of the posterior wall of the left atrium, above the base of the posterior mitral cusp, associated with numerous Aschoff nodules in the subendocardium. This was called MacCallum's Patch or Triangle, because of its triangular distribution (Fig 1). The etiology was thought to be rheumatic. Today, this lesion is considered to be traumatic in origin, caused by the abnormally directed regurgitant stream of mitral regurgitation impinging upon the posterior wall of the left atrium, resulting in the formation of a systolic pocket. Our investigation of the parietal endocardium in acute rheumatic carditis, demonstrated the presence of acute parietal endocarditis, with a reaction similar to that present in the valves [2]. A separate examination of the left atrial endocardium above the base of the posterior mitral cusp, showed the presence of prominence and palisading of the endocardial cells (Fig. 1), with irregular outpouchingprotrusion of the endocardial surface. Our investigation of the parietal endocardium in acute rheumatic carditis, demonstrated the presence of acute parietal endocarditis, with a reaction similar to that present in the valves [2]. A separate examination of the left atrial endocardium above the base of the posterior mitral cusp, showed the presence of prominence and palisading of the endocardial cells (Fig. 1), with irregular outpouching-protrusion of the endocardial surface. There were raised, irregular edematous nodules of the endocardium (Fig. 2), with an infiltration of elongated histiocytes, fibroblasts and nongranular cells (Fig. 3) and macrophages. There was evidence of neovascularization and the formation of collagen fibrils. An increase, was noted, in the smooth muscle components of subendocardium. An endocardial nodule (Fig. 4) showed organization of fibrinoid necrosis. This finding supports the postulate that the endocardial fibrosis results from the organization of the acute rheumatic process.

A similar reaction was present in the mitral valve (Fig. 5). The cusp was edematous, with an infiltration of fibroblasts, elongated histiocytes and nongranular cells. Neovascularization was present with periarteriolar fibrosis and a nongranular cell infiltration (Fig. 6).

We propose that the pathogenesis of MacCallum's Triangle is initiated by the rheumatic process, which undergoes organization and fibrosis. The localization of the lesion to the posterior wall of the left atrium is determined by the traumatic impingement upon it by the regurgitant stream of mitral regurgitation.

References:

[1] MacCallum, WG (1924): Rheumatic lesions of the left auricle of the heart. Bull Johns Hopkins Hosp.,: 35, 329

[2] Siew, S (2008): Rheumatic Heart Disease Revisited: Acute Parietal Endocarditis. Another Piece of the Unsolved Puzzle. MicroscoMicranal. 1552 


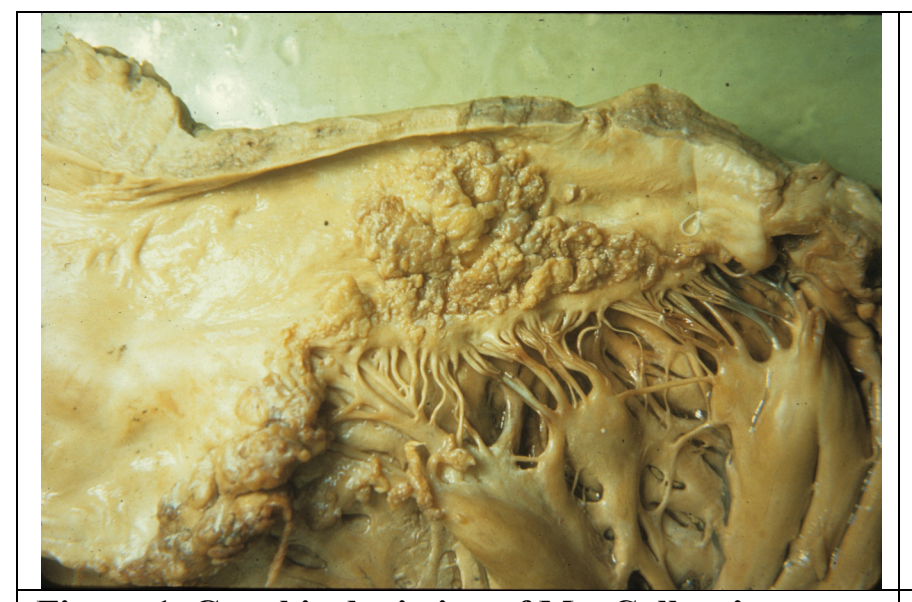

Figure 1. Graphic depiction of MacCallum's Triangle. Spreading vegetations of subacute bacterial endocarditis

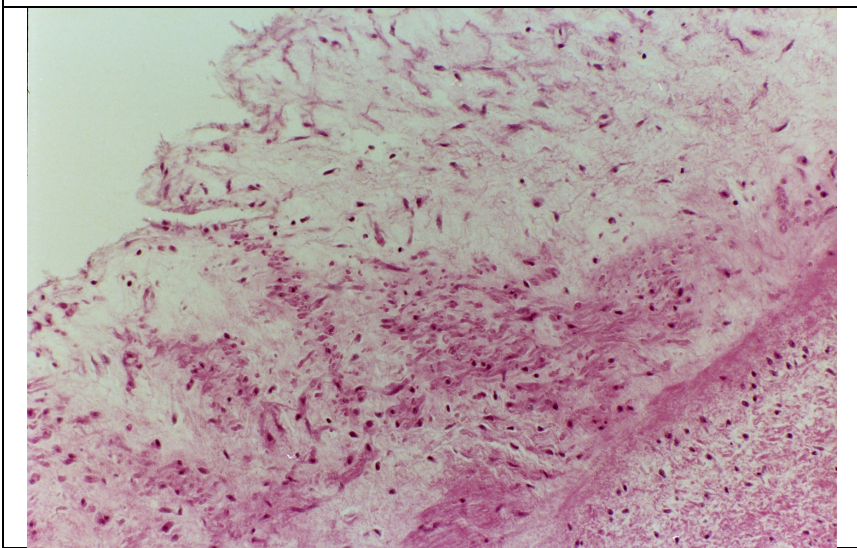

Figure 3. Endocardial nodule infiltration of elongated histiocytes and fibroblasts (original magnification 400x)

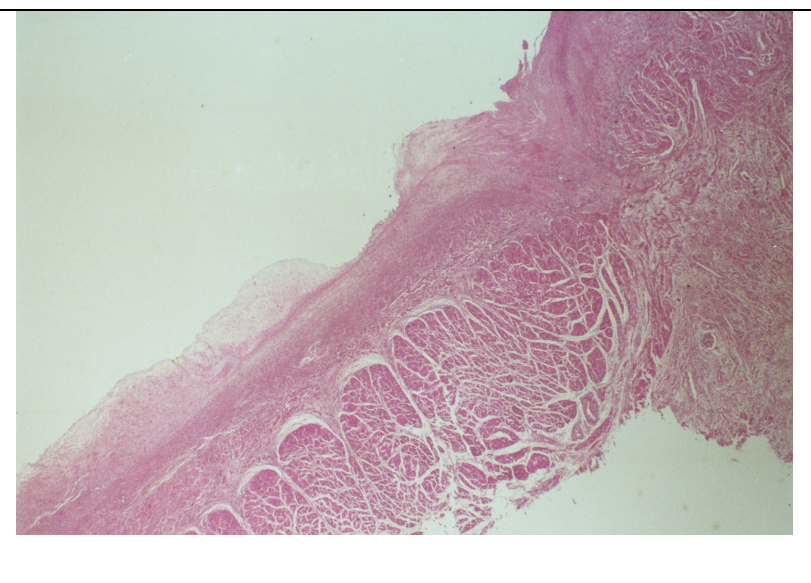

Figure 2. Raised, irregular edematous nodules on the endocardium (original magnification 100x)

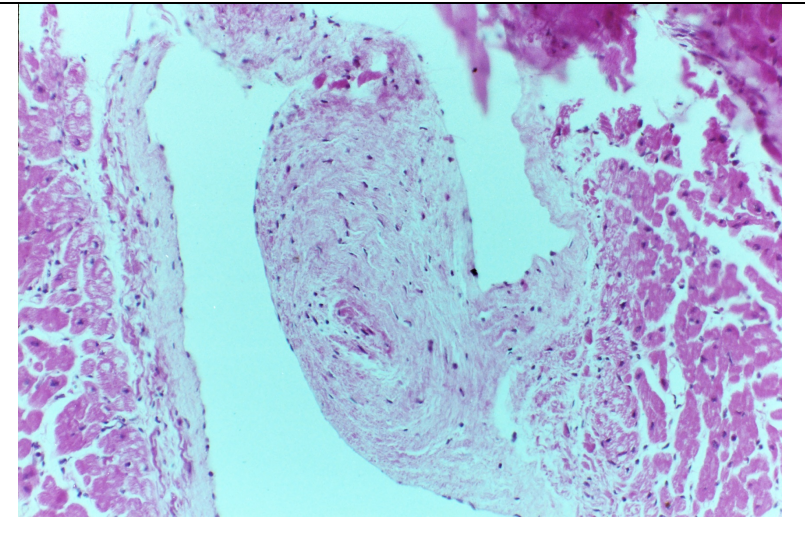

Figure 4. Fibrinoid necrosis in endocardial nodule (original magnification 200x)

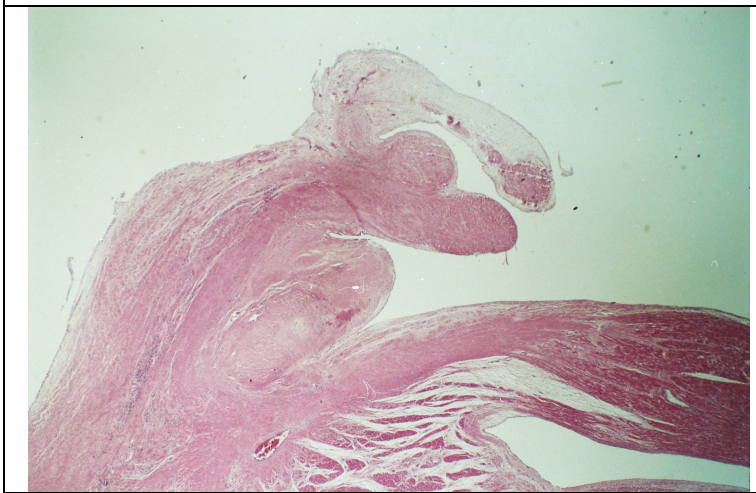

Figure 5. Mitral valve edematous cusp (EC); papillary muscle (PM); chordae tendinae (CT) (original magnification 20x)
Figure 6. Mitral valve - vascularization (V) (original magnification 200x) 